\title{
Threaded cortical bone dowels for lumbar interbody fusion: technique and short-term results
}

\section{Bryan Barnes, M.D., Mark R. McLaughlin, M.D., Barry Birch, M.D., Gerald E. Rodts, Jr., M.D., and Regis W. Haid, Jr., M.D.}

Department of Neurosurgery, Emory Clinic, Atlanta, Georgia; and Department of Neurosurgery, Mayo Clinic, Scottsdale, Arizona

The authors retrospectively reviewed a series of cases involving mechanical low-back or disogenic pain; 35 patients underwent lumbar interbody fusion in which threaded cortical bone dowels (TCBDs) were placed to treat degenerative disc disease.

The series was composed of 18 females, and 17 males whose mean age was 46 years (range 17-76 years). There were nine smokers in the group. All patients presented with symptoms consistent with mechanical low-back or discogenic pain, and magnetic resonance imaging--documented degenerative changes and disc collapse greater than 50\%, as compared with the adjacent normal-appearing level, were confirmed. Twenty-three patients underwent a posterior lumbar interbody fusion (PLIF) procedure for placement of the TCBD, whereas 12 underwent an anterior lumbar interbody fusion (ALIF) procedure for placement of the TCBD. In all patients undergoing PLIF procedures pedicle screw and rod constructs were used without posterolateral fusion except one. In all cases of ALIF except one TCBDs were used as "stand-alone" devices without supplemental fixation. All TCBDs were packed with morselized cancellous autograft prior to implantation. The success of fusion was determined at follow-up intervals and was defined as: the absence of lucency around the TCBD; an increase in subchondral endplate sclerosis; and the presence of bridging bone incorporating the anterior bone graft as demonstrated on static lumbar radiographs and/or computerized tomography scans. Stability was also determined by an absence of movement on dynamic lumbar radiographs. The degree of lumbar lordosis at the diseased level was measured immediately postoperatively and compared with the change in lordosis at follow up. Outcomes were assessed using a modified Prolo outcome scale and rated as excellent, good, fair, or poor. Excellent and good outcomes were considered satisfactory; fair or poor outcomes were considered unsatisfactory.

In 27 patients radiographic and clinical follow-up results were considered adequate (nine ALIF and 18 PLIF patients). The mean follow-up duration was 7.9 months. Overall satisfactory outcome was 70\%: a $77 \%$ satisfactory outcome in PLIF patients and a 55\% in ALIF patients. Osseous fusion was present in $94 \%$ of the patients in the PLIF group and in $33 \%$ of those in the ALIF group. Complications included one L-5 nerve root injury and two postoperative wound infections, all in patients who underwent PLIF; 
there was also a case of breakout of one implant at 8 months postoperatively. The degree of vertebral body angulation measured at last follow up compared with the measurement obtained immediately postoperative was $3.4^{\circ}$ of kyphosis in the ALIF group and $3.1^{\circ}$ of kyphosis in the PLIF group, which represented an $11 \%$ and $9 \%$ loss of lordosis, respectively.

Preliminary results indicate that there is a dramatically higher fusion rate in PLIF compared with ALIF procedures in which TCBDs are used. There is a corresponding trend seen in patient outcomes, but no distinct difference seems apparent in terms of restoration of lordosis when performing either procedure. The results suggest that TCBDs may best be used in PLIF procedures in conjunction with pedicle screws and rod constructs. Moreover, in patients in whom TCBDs and supplemental tension band constructs are used fusion rates appear to be comparable with those reported in other series but at a faster rate (94\% at 7.9 months mean follow up). Longer follow-up periods and a larger series of patients are needed to confirm these preliminary observations.

\section{Key Words * spinal fusion * degenerative disc disease * spinal stabilization * cages * threaded cortical bone dowels}

Techniques for lumbar interbody fusion have evolved rapidly over the last decade because of recent advances in instrumentation. The development of the interbody prosthesis began in veterinary medicine in the 1970s to achieve cervical fusion in horses with cervical spondylosis,[13] and the original device was a cortical bone dowel similar to a Cloward-type fusion. This led to the development of threaded interbody metallic devices that were investigated and cleared by the Food and Drug Administration for use in humans on a limited basis. Subsequently, there has been a large resurgence of procedures in which interbody fusion is performed. A wide array of devices are available for interbody fusion at all levels of the spine. Although fusion rates and outcomes in procedures in which threaded cortical bone dowels (TCBDs) are used in the cervical region are well documented, little has been reported regarding TCBDs in the lumbar region. In this report we detail our preliminary results with TCBDs for lumbar interbody fusions and review the literature regarding interbody devices.

\section{CLINICAL MATERIAL AND METHODS}

Between June 1997 and December 1998, anterior or posterior lumbar interbody fusion (ALIF or PLIF) procedures were performed in 35 patients by two surgeons who used TCBDs (Regeneration Technologies, Alachua, FL) to treat mechanical low-back or discogenic pain. The series was composed of 18 females and 17 males with a mean age of 46 years (range 17-76 years). There were nine active cigarette smokers in the series. Patient data were collected by chart review and clinical follow-up examination. Immediate postoperative static radiographs and follow-up static and dynamic radiographs were reviewed to determine changes in lordosis as well as the success of fusion and spinal stability. In some cases, computerized tomography (CT) scanning was used for supplemental verification of plain radiographic findings. All patients presented with symptoms consistent with mechanical low-back pain or discogenic pain, with magnetic resonance imaging--confirmed degenerative changes and disc collapse greater than 50\% compared with the adjacent normal-appearing vertebral level. Twenty-three patients underwent PLIF and 12 underwent (ALIF) for the placement of the TCBD implant. In all patients except one undergoing PLIF, pedicle screw and rod constructs were used without posterolateral fusion. In all ALIF procedures except one, TCBDs were used as "stand-alone" devices without posterior tension band fixation. All TCBDs were packed with morselized cancellous autograft prior to implantation. Outcomes assessed using a modified Prolo[8] scale were rated as excellent, good, fair, or poor. Excellent and good 
outcomes were considered satisfactory; fair or poor outcomes were considered unsatisfactory.

Operative criteria for ALIF were intractable back pain that did not respond to nonoperative management; minimal or no radicular pain; significant single disc degeneration observed on magnetic resonance imaging with disc space collapse greater than 50\%; and concordant pain provocation as demonstrated on discography in some cases. Operative criteria for PLIF were the same with the addition of significant posterior disease such as lateral recess or foraminal stenosis.

In the PLIF group, the levels at which implants were inserted were L3--4 (one case), L4--5 (11 cases), L5--S1 (eight cases), transitional (L-6)--S1(one case), L4--S1 (two cases [two-level implant]); and the ALIF group, L5--S1 (10 patients), and L4--S1(two patients [two-level implant]). Fusion was determined at follow-up intervals and was defined as: the absence of lucency around the TCBD; an increase in subchondral endplate sclerosis; and the presence of bridging bone incorporating the anterior bone graft as demonstrated on static lumbar radiographs (Fig. 1).

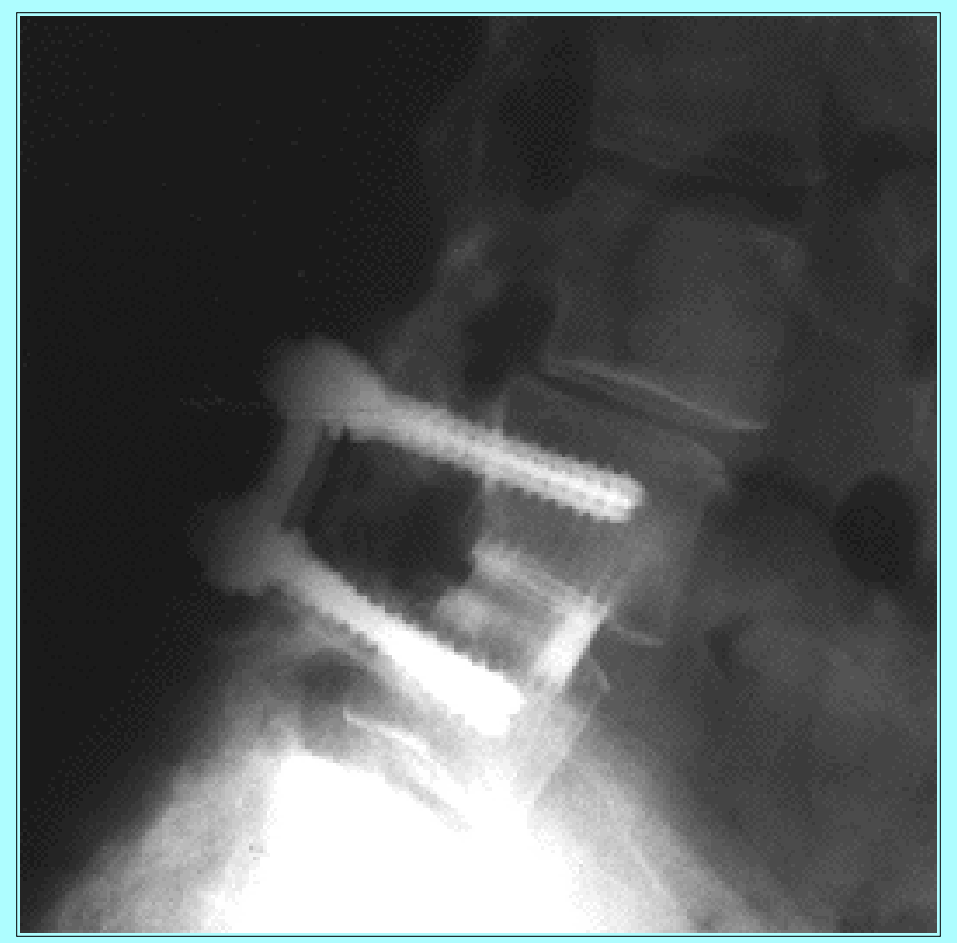

Fig. 1. One-year follow-up plain lateral radiograph obtained in a patient who underwent a PLIF procedure with threaded cortical bone dowels and supplemental pedicle screw fixation at L4--5. There is the absence of lucency around the TCBD and evidence of bone bridging posteriorly. This patient also had asymptomatic anterior spondylolisthesis of L-3 on L-4, which appeared stable on dynamic radiographs.

In some cases, plain and myelographic CT scanning was used to supplement plain radiographic findings (Fig. 2). Stability was also defined by an absence of movement on dynamic lumbar radiographs. The radiographic determination of fusion and stability was confirmed in all cases by both the senior author (R.W.H.) and an attending radiologist. 


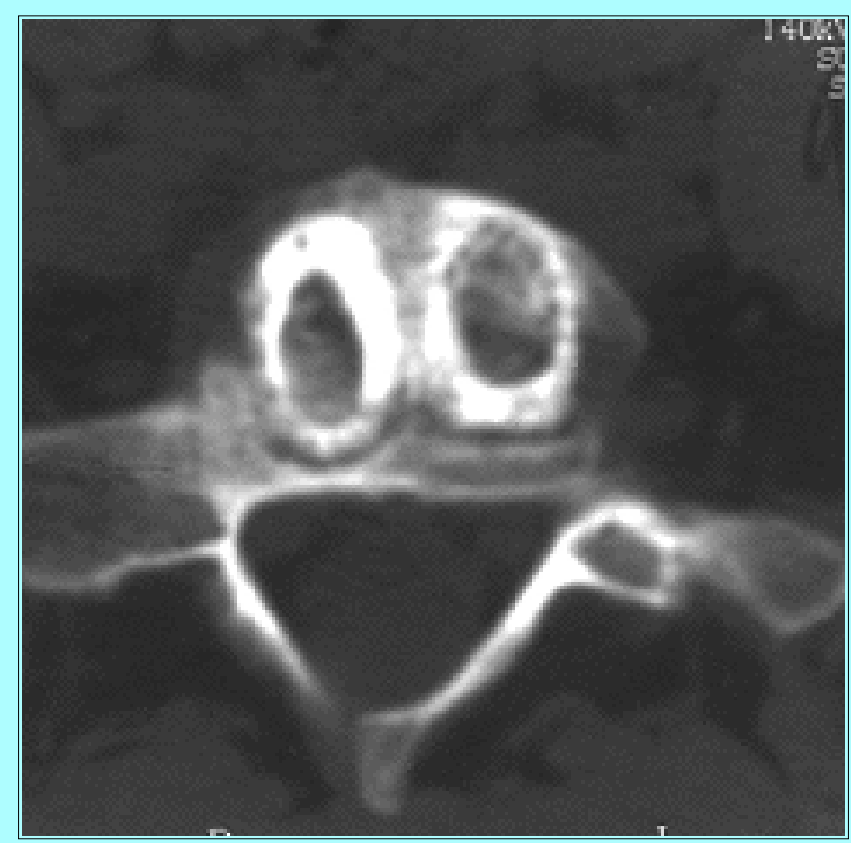

Fig. 2. Eight-month follow-up axial CT scan demonstrating early fusion at L4--5 in a patient who underwent an ALIF procedure, with incorporation of the threaded cortical bone dowel anterior to the graft. There is a slight lucency posterior to the graft, but the majority of the graft appears to be well incorporated.

Angulation was determined using a modified Cobb technique to measure a line parallel to the superior endplate of the superior vertebral body and a line parallel to the inferior endplate of the inferior vertebral body. Angulation as observed on immediate postoperative films was measured and compared with vertebral body angulation determined on follow-up films.

Outcomes were measured using a modified Prolo[8] scale as being excellent, good, fair, or poor, based on clinical follow-up notes. In patients with excellent outcomes a significant improvement in pain status was shown, they were participating fully in their premorbid activities and/or working full time, and they required minimal or no analgesic/narcotic substances. In patients with good outcomes an improvement in pain status was shown, they were working parttime or participating intermittently in premorbid activities, and they required diminishing narcotic/analgesic substances compared with preoperative status. In patients with fair outcomes a mild improvement in pain status was shown, their participation in premorbid activities or work was significantly dimished, and they still required the same amount of narcotic/analgesic medications. In patients with poor outcomes pain status was unchanged or worse than their premorbid status, they were not working or participating in premorbid activities, and they required the same or increased amounts of narcotics/analgesic medications.

\section{RESULTS}

In 27 patients adequate clinical and radiographic follow-up data were obtained, including nine (75\%) of 12 patients in the ALIF group and 18 (78\%) of the 23 in the PLIF group. There were no significant differences in age, sex, or smoking characteristics between the initial 35 patients and those included in the results (analysis of variance, $\mathrm{p}<0.05$ ). There were also no significant differences in age, sex, or smoking characteristics between the ALIF and PLIF groups $(\mathrm{p}<0.05)$. The overall mean duration of follow up was 7.9 months. The mean duration of follow up in PLIF patients was 7 months, whereas in the ALIF patients it was 10.4 months. 
Overall outcome was 70\% satisfactory: a 77\% satisfactory outcome in PLIF patients and a 55\% satisfactory outcome in ALIF patients.

The overall fusion rate in patients who underwent ALIF procedures was 33\%, whereas it was 94\% (17 of the 18 cases with adequate follow up) in those who underwent PLIF.

The change in angulation was $3.4^{\circ}$ of mean kyphosis in ALIF patients and $3.11^{\circ}$ of kyphosis in PLIF patients, representing a mean $11 \%$ and $9 \%$ loss of lordosis, respectively. The change in angulation as determined on immediate postoperative films and follow-up films was $8^{\circ}$ of kyphosis to $5^{\circ}$ of lordosis in ALIF patients, $8^{\circ}$ of kyphosis to $8^{\circ}$ of lordosis in PLIF patients, respectively.

There were four complications: one L-5 nerve root injury, which resolved with observation; two postoperative wound infections, which resolved with operative debridement, irrigation, and intravenously administered antibiotics; and one case of lateral breakout of one implant, which was detected on a CT scan 8 months postoperatively (Fig. 3).

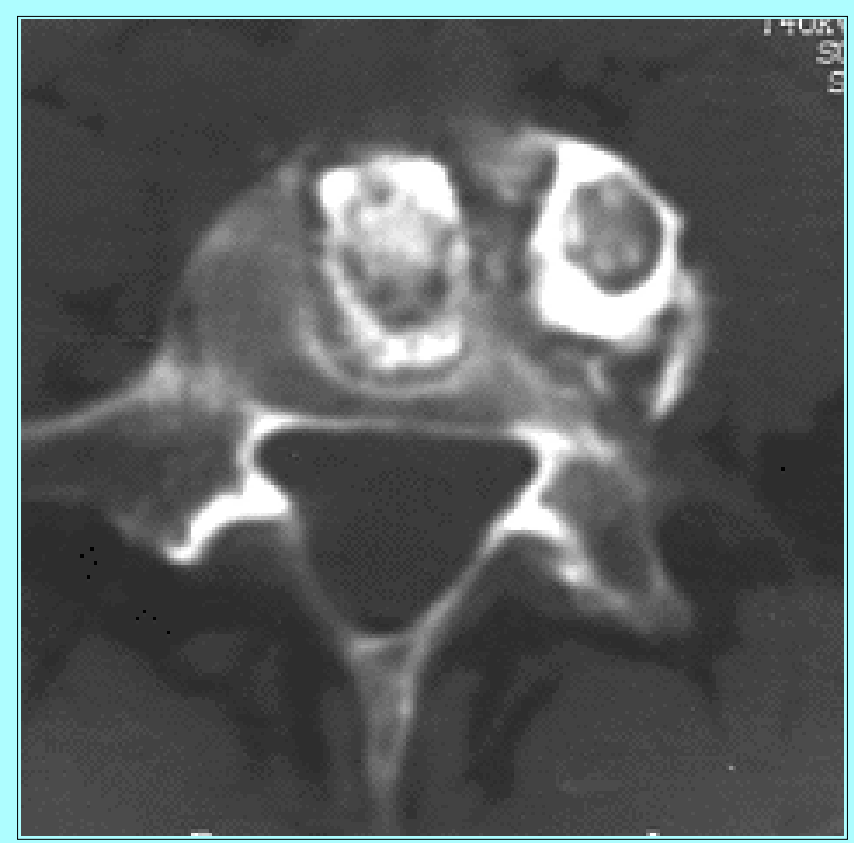

Fig. 3. Axial CT scan demonstrating clear nonunion, with lucency surrounding the threaded cortical bone dowel. Note that there is evidence of left-sided lateral breakout of left dowel. The patient was asymptomatic despite this finding.

\section{DISCUSSION}

Among lumbar interbody fusion devices, titanium cages have been established as providing a reliably high fusion rate.[4-6,9-11,15] There are numerous types of cages available including horizontal cylinders, vertical rings, and open boxes. Little biomechanical information is available regarding the stability of vertical rings and open boxes.[14] Horizontal cylinders such as the BAK cage, Ray, and impacted carbon cages provide excellent segmental stability, as has been shown in both animal and human cadaver studies. $[2,3,5,7,12]$ Investigators in prospective multicenter studies of the BAK device found overall fusion rates of $85.6 \%, 90.6 \%$, and $98.3 \%$ at 1,2 , and 3 years postoperatively, respectively, with significant improvement in pain and function among the patient population (Unpublished datat). There was a 96\% fusion rate at 2-year follow up reported in the Ray[9] cage series, with $86 \%$ of patients obtaining satisfactory clinical relief of their back or radicular pain. Agazzi, et al.,[1] have reported a 90\% 
fusion rate at a mean 28-month follow up when using the impacted carbon cages.

When compared with titanium cages, TCBDs may have the advantage of allowing easier assessment of radiographic fusion and may be more amenable to surgical revision. Moreover, TCBDs have a modulus of elasticity similar to the native bone in the area of implantation as opposed to titanium and carbon fiber cages. Theoretically this may provide a higher fusion rate by minimizing stress shielding of the graft and decreasing the degree of subsidence.

It is our opinion that the reason for increased incidence of fusion in patients undergoing PLIF with supplemental tension band fixation compared with those undergoing ALIF in which "stand-alone" devices were used is related to increasing the compression and therefore the stress on the graft (Wolff's law), although we have no objective data to support this contention. Additionally, given such a short follow-up period, there is the possibility that TCBDs implanted in the ALIF procedure may simply take longer to fuse.

\section{CONCLUSIONS}

There was a markedly higher incidence of fusion in patients who underwent PLIF with TCBDs and pedicle screw fixation than in those who underwent ALIF with TCBDs alone. This difference in fusion rate correlated with improved clinical outcome in our series of patients, as evidenced by a $20 \%$ higher satisfactory outcome rate in those in whom a posterior rather than an anterior approach was performed. There appears to be no trend toward differential postoperative angulation with restoration of lordosis based on approach.

Although the mean duration of follow up was less than 1-year, three of our six ALIF patients in whom fusion had not yet been achieved had a 1-year or greater follow up. This indicates what we believe to be a genuine trend toward suboptimal outcomes when using bone dowels as stand-alone anterior implants. As a corollary, the use of TCBDs with supplemental pedicle screw fixation is associated with a high and rapid fusion rate (94\%) comparable to previously published rates with titanium interbody cages.

These results, although preliminary, reflect favorably on our initial experiences with the PLIF procedure in which TCBDs and pedicle screws are used. A longer follow-up period and a greater number of patients are needed to distinguish further the potential advantages and disadvantages of bone dowels compared with other lumbar interbody fusion techniques.

\section{References}

1. Agazzi S, Reverdin A, May D: Posteror lumbar interbody fusion with cages: an independent review of 71 cases. J Neurosurg (Spine 2) 91:186-192, 1999

2. Brantigan JW, Steffee AD, Geiger JM: A carbon fiber implant to aid interbody lumbar fusion: mechanical testing. Spine 16 (Suppl 6):S277-S282, 1991

3. Brodke DS, Dick JC, Kunz DN, et al: Posterior lumbar interbody fusion. A biomechanical comparison, including a new threaded cage. Spine 22:26-31,1997

4. Dickman CA: Internal fixation and fusion of the lumbar spine using threaded interbody cages. BNI Q 13:4-25, 1997 
5. Kuslich SD, McAfee PC, Regan JF: Spinal instrumentation, in Regan JF, McAfee PC, Mack MF (eds): Atlas of Endoscopic Spine Surgery. St. Louis: Quality Medical, 1995, pp 293-331

6. Mahvi DM, Zdeblick TA: A prospective study of laparoscopic spinal fusion. Technique and operative complications. Ann Surg 224:85-90, 1996

7. Oxland TR, Lund T, Jost B, et al: The relative importance of vertebral bone density and disc degeneration in spinal flexibility and interbody implant performance. An in vitro study. Spine 21:2558-2569, 1996

8. Prolo DJ, Oklund SA, Butcher M: Toward uniformity in evaluating results of lumbar spine operations. A paradigm applied to posterior lumbar interbody fusions. Spine 11:601-606,1986

9. Ray CD: Threaded titanium cages for lumbar interbody fusions. Spine 22:667-680, 1997

10. Regan JJ, McAfee PC, Guyer RD, et al: Laparoscopic fusion of the lumbar spine in a multicenter series of the first 34 consecutive patients. Surg Laparose Endosc

11. Regan JJ, Yuan H, McAfee PC: Laparoscopic fusion of the lumbar spine: minimally invasive spine surgery. A prospective multicenter study evaluating open and laparoscopic lumbar fusion. Spine 24:402-411, 1999

12. Riley LH III, Eck JC, Yoshida H, et al: Laparoscopic assisted fusion of the lumbaosacral spine. A biomechanical and histologic analysis of the open versus laparoscopic technique in an animal model. Spine 22:1407-1412, 1997

13. Wagner PC, Grant BD, Bagby GW, et al: Evaluation of cervical spinal fusion as a treatment in the equine "wobbler" syndrome. Vet Surg 8:84-89, 1979

14. Weiner BK, Fraser RD: Spine update--lumbar interbody cages. Spine 23:634-640, 1998

15. Zucherman JF, Zdeblick TA, Bailey SA, et al: Instrumented laparoscopic spinal fusion: Preliminary results. Spine 20:2029-2035, 1995

Manuscript received October 1, 1999.

Accepted in final form December 6, 1999.

Address reprint requests to: Bryan Barnes, M.D., Department of Neurosurgery, Emory Clinic, 1365-B Clifton Road, N.E., Suite B2200, Atlanta, Georgia 30322. 